\title{
Why should we bother diagnosing cystic fibrosis in adult patients?
}

\section{Denis Hadjiliadis*}

Assistant Professor of Medicine Pulmonary, Allergy and Critical Care University of Pennsylvania

\section{Editorial}

Cystic fibrosis (CF) is the most common life limiting disease in Caucasian patients. Over the past decades improvements in patient care have led to significantly improved survival [1]. As a result approximately $50 \%$ of patients with CF are now adults [1].

In recent years, it has become obvious that a small but significant percentage of patients do not get diagnosed with cystic fibrosis until adulthood [1]. Most of them have only some manifestations of cystic fibrosis; they usually do not have pancreatic insufficiency, but frequently have some or all of the following: bronchiectasis, chronic sinusitis, recurrent acute pancreatitis, male infertility (absence of the vas deferens) [2-5]. Diagnosis in such patients requires careful history, often dating back to childhood, physical examination and laboratory evaluation. In addition, specialized tests with careful interpretation are also needed; these include sweat testing, genetic testing for CF and nasal potential difference testing (when available) $[4,5]$. These tests frequently have significant costs and patients might have significant travel and waiting times for their appointments at specialized cystic fibrosis centers. Until recently there was not enough information on the clinical course of patients with CF diagnosed in adulthood and whether specialized expert care is beneficial.

However, recent studies have suggested that patients diagnosed with CF in adulthood can have severe disease, especially pulmonary disease, despite atypical (borderline) diagnostic tests [2,3,6]. In addition, patients diagnosed in adulthood can experience lung function decline over the years $[2,3,6]$. These findings suggest that adult diagnosed patients have severe disease. More importantly, another study from National Jewish suggested that care in an adult CF center was associated with improvements in lung function early after diagnosis [2]. This was also found in a smaller case control study at the University of Pennsylvania [6]. Accurate diagnosis can help provide access to appropriate medications (for example, dornase-alpha for example is beneficial in $\mathrm{CF}$, but not in non-CF bronchiectasis; other medications like tobramycin for inhalation solution or aztronam lysinate for inhalation are much easier or only possible to obtain if the patient has a diagnosis of CF). Specialized care in CF centers not only encompasses medical care with the appropriate medications, but it includes multidisciplinary care from many healthcare professionals. Specialists in nutrition, airway clearance, physical therapy and exercise, as well as social workers familiar in navigating the complicated insurance landscape are the likely reasons for the improvement in lung function seen at CF centers. Furthermore, specialists in diabetes, ENT, hepatology and gastroenterology can provide expert care for other problems patients with CF might face. Finally, patients might be able to provide significant genetic information to their families and thus help them avoid or prepare for future children born with cystic fibrosis.

Universal screening might make adult diagnosis almost obsolete in the future (even patients that have borderline diagnostic might be followed at a CF center and utilize its expertise as needed). However, there are still many patients who remain undiagnosed late into their adulthood, because CF is still considered a pediatric disease and because of atypical symptoms (or only disease in some and not all organs frequently affected by $\mathrm{CF}$ ). Education to all the appropriate specialties that might treat patients with undiagnosed CF will help identify such patients.

In summary, patients diagnosed with CF in adulthood can have atypical diagnostic tests, but severe disease [2-5]. Their disease can be progressive and be associated with morbidity and mortality $[2,3,6]$. However, care in a specialized center is associated with improvement of lung disease (a major source of morbidity and mortality). Based on these data we think that appropriate diagnosis of CF in adults and care in specialized centers is warranted. It is likely that it will help improve the morbidity and mortality of CF in this group of patients. Longerterm studies will be able to prove or refute this point.

\section{References}

1. Cystic Fibrosis Foundation (2010) Cystic Fibrosis Foundation Patient Registry: 2009 annual data report to the center directors. Bethesda, MD: Cystic Fibrosis Foundation.

2. Nick JA, Chacon CS, Brayshaw SJ, Jones MC, Barboa CM, et al. (2010) Effects of gender and age at diagnosis on disease progression in long-term survivors of cystic fibrosis. Am J Respir Crit Care Med 182: 614-626.

3. Keating CL, Liu X, DiMango EA (2010) Classic respiratory disease but atypical diagnostic testing distinguishes adult presentation of cystic fibrosis. Chest 137: 1157-1163.

4. Gilljam M, Ellis L, Corey M, Zielenski J, Durie P, et al. (2004) Clinica Manifestations of Cystic Fibrosis Among Patients with Diagnosis in Adulthood. Chest 126: 1215-1224.

5. Hubert D, Fajac I, Bienvenu T, Desmazes-Dufeu N, Allaffi M, et al. (2004) Diagnosis of cystic fibrosis in adults with diffuse bronchiectasis. J Cyst Fibros 3: 203.

6. Hadjiliadis D, Ferrin M, Imbesi G, Stephen MJ, Lipson DA et al. (2008) Clinical and Genetic Manifestations of Cystic Fibrosis Patients Diagnosed as Adults. Pediatr Pulmonol 43: 407.
Corresponding author: Denis Hadjiliadis, Assistant Professor of Medicine Pulmonary, Allergy and Critical Care, University of Pennsylvania, Philadelphia, PA 19104, Tel: 215-615-3871; Fax: 215-614-0869; Email: Denis.Hadjiliadis@uphs. upenn.edu

Received September 26, 2011; Accepted October 17, 2011; Published October 19, 2011

Citation: Hadjiliadis D (2011) Why should we bother diagnosing cystic fibrosis in adult patients? J Pulmonar Respirat Med 1:e102. doi:10.4172/2161-105X.1000e102

Copyright: (c) Hadjiliadis D. This is an open-access article distributed under the terms of the Creative Commons Attribution License, which permits unrestricted use, distribution, and reproduction in any medium, provided the original author and source are credited. 